DOI: https://doi.org/10.29105/gmjmx15.28-5

Artículos

\title{
PREDICTORES DE LAS TENDENCIAS MORALES ASOCIADAS AL USO DE FACEBOOK ${ }^{1}$
}

\author{
Rolando Pérez Sánchez \\ Universidad de Costa Rica, Costa Rica \\ Gloriana Giusti Mora \\ Universidad de Costa Rica, Costa Rica \\ Karina Soto Chavarría \\ Universidad de Costa Rica, Costa Rica \\ Autor para correspondencia: Rolando Pérez Sánchez, rolarez@gmail.com
}

\section{Resumen}

Este artículo es un aporte al estudio del procesamiento moral del uso de Facebook desde el punto de vista de la cognición social. La investigación tuvo por objetivo identificar algunos predictores de las tendencias morales de uso, especificamente aquellas denominadas por la investigación antecedente como hedonista y eudaimónica, de esta red social. El énfasis se concentró en la medición de las posibles asociaciones entre un tipo de comportamiento auto-presentativo y otro prosocial y la presencia de las tendencias hedonistas y eudaimónicas. Se recurrió a un cuestionario auto-aplicado, administrado a 122 estudiantes universitarios (62.5\% mujeres y edad promedio 20.77, DT: 6.85). Los resultados mostraron una asociación negativa entre el comportamiento prosocial y las tendencias hedonistas, así como otra asociación positiva entre el comportamiento de uso prosocial y la tendencia moral eudaimónica. Se discuten estos resultados identificando sus alcances y señalando la necesidad de mayor investigación futura.

Palabras clave: Facebook, razonamiento moral, intuiciones morales, altruismo, redes sociales.

\begin{abstract}
This article aims to contribute to the study of the moral processing of the use of Facebook from a social cognition perspective. The goal of this research was to identify some predictors of the hedonistic and eudaimonic use tendencies of this social network. We focused on the measurement of possible associations between a self-presentative and a prosocial type of behavior and the presence of hedonistic and eudaimonic tendencies. In order to do this, we used a self-applied questionnaire that was administered to 122 university students (62.5\% women, mean age 20.77, SD: 6.85). Results showed a negative association between prosocial behavior and hedonistic
\end{abstract}

\footnotetext{
${ }^{1}$ La investigación fue financiada por la Vicerrectoría de Investigación de la Universidad de Costa Rica.

Global Media Journal México 15(28). Enero - junio 2018. Pp. 66-77.
} 
tendencies, and a positive association between prosocial behavior and eudaimonic moral tendency. These results are discussed pointing to need for more research in this topic.

Keywords: Facebook, moral reasoning, moral intuitions, altruism, social networks.

Recibido: 9/04/2018

Aceptado: 31/05/2018

\section{Introducción}

Este artículo tiene como interés general contribuir al estudio de los mecanismos de la cooperación, la coordinación de la acción, el disfrute, la prosocialidad y la empatía, que se pueden generar a partir del uso de las redes sociales, en este caso Facebook.

La discusión actual sobre los usos psicosociales toma como base conceptual el modelo de dos factores del entretenimiento (Vorderer y Reinecke, 2015). Hasta ahora el estudio de la psicología de los usos y efectos de los medios se ha basado fundamentalmente en la idea de que los usuarios tienen una motivación hedonista en el consumo del contenido mediático, lo que implica la búsqueda del placer (Vorderer, Klimmt y Ritterfeld, 2004). Según esta perspectiva, las personas se orientan a usar los medios con el objetivo de regular sus estados de ánimo, buscando centralmente aquellos contenidos que les permitan orientarse hacia afectos positivos (Zillmann, 1988; Bryant, Eweldosen y Cantor, 2003, Vorderer y Reinecke, 2015).

Producto del cuestionamiento a la reducción del uso mediático a un modelo hedonista (Tamborini, 2013), se ha incorporado en la investigación un debate entre el modelo hedonístico y el modelo eudaimónico del uso mediático (Eden, Grizzard y Lewis, 2013; Reinecke, Vorderer y Knop, 2014). Estas conceptualizaciones han sido incluidas dentro de un "modelo de dos factores" del disfrute (Vorderer, 2011; Vorderer y Reinecke, 2015), pretendiendo ir más allá de una concepción hedonística del consumo de los medios, y agregándole a su vez, un componente eudaimónico, que plantea que las personas pueden utilizar los medios con fines de apreciación, es decir, con el propósito de evaluar y reflexionar los contenidos mediáticos (Oliver y Bartsch, 2010).

El modelo eudaimónico deviene de la ética aristotélica, que señala que la consecución de la felicidad puede ser alcanzada a través de la virtud y de la afirmación de un sistema de creencias y convicciones morales, condición que se ha denominado eudaimonía (Aristóteles, 1985; Eden et al., 2013). Esta concepción, aplicada al campo de la psicología de los medios de comunicación, implica un uso mediático orientado por criterios morales asociados al altruismo, la prosocialidad y la justicia, 
centrados en la reflexividad y apreciación crítica de los contenidos aportados por el medio específico.

Los criterios morales antes mencionados, al igual que en otros contextos de evaluación moral, operan según un modelo dual, en donde la persona puede recurrir a intuiciones o heurísticos morales (nivel automático) o al razonamiento moral (nivel controlado) (Weber, Popova y Mangus, 2013).

En el caso de Facebook, tema central de este estudio, no se ha investigado aún sobre los usos eudaimónicos y hedonistas asociados al mismo, pero se han realizado estudios sobre el entretenimiento en el ámbito fílmico o literario que permiten orientar y conceptualizar las hipótesis para el caso de las redes sociales.

En esa línea investigativa, Oliver y Raney (2011) estudiaron cómo las personas no sólo buscan el placer como motivador de consumo de material fílmico (preocupación hedónica), sino que también pueden optar por la búsqueda de la verdad en el uso de este (preocupación eudaimónica). Como parte de sus resultados, los investigadores encontraron una coexistencia de motivaciones hedonísticas y eudaimónicas. La primera de estas se orienta a la distracción y a la diversión, es decir, a tendencias más alegres y lúdicas, mientras que la segunda se dirige a la búsqueda de contenido de "significativo", vinculado a tendencias más contemplativas y reflexivas.

En otro estudio, Wirth, Hofer y Schramm (2012) examinaron las tendencias eudaimónicas durante la exposición de una película triste y a la vez significativa en cuanto su contenido, con el objetivo de valorar la presencia de un tipo de entretenimiento eudaimónico. Se encontró que estas tendencias en los participantes no dependen de si el final es o no feliz, sino más bien de lo que la trama de la película transmite en relación a la vida y lo que esta debería o podría llegar a ser. Wirth et al. (2012) consideran que estas reacciones devienen en un afecto positivo y en el sentimiento de crecimiento personal.

Lewis, Tamborini y Weber (2014), en un estudio experimental investigan el procesamiento de diferente material narrativo encontraron que la presencia de mayor apreciación está asociada con un mayor tiempo de evaluación del material narrativo. Bartsch y Hartmann (2017), en consonancia con el modelo dual del procesamiento mediático, encontraron que los filmes que evocan mayor apreciación demandan más recursos cognitivos, mientras que los filmes de suspenso son los que requieren más recursos afectivos.

A pesar de que las investigaciones mencionadas dan información sobre el entretenimiento asociado a usos hedonistas y eudaimónicos, como se mencionó anteriormente, parece que no se ha realizado aún algún estudio asociado al uso de Facebook, lo que deja ver la necesidad de investigaciones en esta línea.

Los medios como entretenimiento tienen el potencial de proveer un contexto que propicia una conducta auto-determinada

Global Media Journal México 15(28). Enero - junio 2018. Pp. 66-77. 
que puede facilitar la satisfacción de distintas necesidades humanas y el crecimiento humano (Reinecke, Vorderer y Knop, 2014). Además, es en las interacciones humanas y dependiendo del escenario en que estas se desenvuelvan, que la comunicación es capaz de ser parte del bienestar en las personas (Muñiz, 2015). Es por las razones anteriores que se considera relevante la realización de este estudio, pues indagar sobre algunos predictores de las tendencias de uso eudaimónico o hedonista del Facebook como medio de entretenimiento e interacción con otros, puede contribuir al conocimiento de esta red social como una potencial herramienta hacia la satisfacción de necesidades y el bienestar de sus usuarios.

Se eligió Facebook como red social de estudio para esta investigación, ya que, según el rastreador de actividad en la web Stat Counter (2018), en Costa Rica entre el año 2017 y 2018 esta es la red social más utilizada en el país, con $64,46 \%$ de uso, mientras que la segunda es Pinterest con $17,3 \%$ y la tercera Youtube con $13,78 \%$. El resto del porcentaje se distribuye entre otras redes sociales. Cabe destacar que estas estadísticas se basan en el acceso a la plataforma a partir de computadoras, tabletas, teléfonos inteligentes y consolas.

En particular interesa estudiar si los comportamientos en Facebook orientados a la autopresentación o a la prosocialidad, el altruismo y la intensidad de uso de Facebook contribuyen a explicar la presencia de una tendencia hedonista o eudaimónica.

Por usos prosociales se entiende aquel tipo de actividad en dicha red social, orientada a interesarse por su grupo de amigos, mostrar apoyo, empatía, así como la participación en grupos preocupados por temas sociales o ambientales. Los usos autopresentativos se orientan, por el contrario, a expresar los estados de ánimo propios, describir las actividades personales que se están realizando en un momento dado, o el mostrar fotos en las que la persona está involucrada.

El altruismo es un tipo de conducta prosocial, que se refiere a la ayuda voluntaria que se da motivada por la preocupación hacia el bienestar y las necesidades de otros. Los motivos principales para llevar a cabo una acción prosocial son la simpatía y las normas o principios internalizados. La simpatía se refiere a la preocupación por otros que se basa en la comprensión de su estado emocional y puede derivarse de la empatía, que es una reacción emocional que surge y coincide con el estado emocional de la otra persona. Por otro lado, cuando se tienen principios o normas internalizadas, en especial cuando están asociados a la importancia de ayudar a los demás, es probable que estas personas lleven a cabo acciones en beneficio de los otros (Carlo y Randall, 2002). Interesa estudiar si la presencia de este tipo de conductas, independientemente de que se lleven a cabo 
mediante la red social Facebook o no, predicen las tendencias hedonistas $\mathrm{o}$ eudaimónicas.

En estudios precedentes se ha encontrado que los usos predominantemente auto-presentativos se asociación con la presencia de un tipo de personalidad narcisística (Gnambs y Appel, 2017), resultados que respaldan el estudio de las tendencias hedonistas y sus consecuencias en la actividad al interior de Facebook. De igual manera, se ha encontrado que un uso activo de esta red social, orientado a exponer sus propias ideas o participar en grupos, se ha asociado en repetidos estudios con un alto bienestar subjetivo (Verduyn, Ybarra, Résibois, Jonides y Kross, 2017). Estos resultados aportan evidencias sobre el uso eudaimónico y la necesidad de su investigación sistemática.

Junto a las conductas de uso y el altruismo se considera relevante la intensidad de uso de Facebook para entender las tendencias hedonistas o eudaimónicas, en tanto puede ser considerado el tiempo de exposición a dicha plataforma como un indicador de involucramiento y disfrute asociado al tipo de uso. Por intensidad de uso se entiende la cantidad de horas semanales que la persona dedica a esta plataforma. Diferentes estudios han encontrado una asociación entre la intensidad de uso de Facebook y las variables como bienestar subjetivo o personalidad. En estudios sobre la relación existente entre el uso de Facebook y variables como la satisfacción con la vida, el narcicismo y la extroversión se han podido encontrar correlaciones positivas entre dichos rasgos de la personalidad y una mayor cantidad o frecuencia de uso de esta red social (Valenzuela, Park y Kee, 2009; Panek, Nardis y Konrath, 2013; Horton, Reid, Barber, Miracle y Green, 2014; Marshall, Lefringhausen y Ferenczi, 2015). Si bien en el presente estudio no se consideran estas variables, estos datos pueden servir como punto de partida para suponer que la intensidad de uso de Facebook está relacionada con el tipo de uso que se haga de la red.

Se consideraron las siguientes hipótesis:

H1. Las tendencias de uso eudaimónico se asociarán a altos puntajes en altruismo.

H2. Las tendencias de uso hedonista se asociarán a bajos puntajes en altruismo.

H3. Una tendencia alta de uso hedonista de Facebook se asociará con bajos puntajes en el uso prosocial y altos en el uso auto-presentativo.

H4. Una auto-evaluación alta del uso eudaimónico de Facebook se asociará con altos puntajes en el uso prosocial y bajos en el uso auto-presentativo.

Global Media Journal México 15(28). Enero - junio 2018. Pp. 66-77. 
Método

\section{Tipo de estudio}

Se trata de un estudio transversal correlacional. La investigación fue sometida a evaluación y aprobada por el Comité Ético Científico de la Universidad de Costa Rica.

\section{Participantes:}

Se recurrió a un muestreo intencional por criterio: 122 estudiantes universitarios, $62,5 \%$ mujeres y una edad promedio de 20,77 (DT: 6,85). Los participantes debían ser usuarios frecuentes de la plataforma de Internet de redes sociales Facebook, visitando al menos una vez al día dicha red.

Los estudiantes participaron voluntariamente en el estudio. El reclutamiento de las personas participantes se hizo por medio de un cartel que se distribuyó en espacio públicos del campus universitario, así como mediante la visita a cursos en donde se les invitaba a colaborar. Cada participante llenó un consentimiento informado donde se le indicó el tratamiento anónimo y confidencial de la información y la ausencia de riesgo evidente al participar en el estudio.

Cada participante llenó un cuestionario que fue diseñado para ser completado en computadora. La actividad se llevó a cabo en un laboratorio dispuesto para tal efecto. La recolección se realizó durante el año 2014 en la Universidad de Costa Rica, en San José, Costa Rica.

\section{Instrumentos y procedimiento}

Para medir las orientaciones prosociales se recurrió a la subescala de altruismo de la Prosocial Tendencies Measure (PTM) de Carlo y Randall (2002), en la versión para Costa Rica de Rivera y Garbanzo (2010). La medida consta de seis ítems, a ser calificados en una escala Likert de cinco puntos, redactados de forma inversa, es decir, bajos puntajes corresponden altos niveles de altruismo. Ejemplos de los ítems es "Creo que si ayudo a alguien en el futuro ellos me deben ayudar", "Una de las mejores cosas de hacer trabajos caritativos es que luce bien en mi curriculum". En el estudio de Rivera y Garbanzo (2010) se obtuvo un coeficiente Alfa de Cronbach de .81. Carlo y Randall (2002) reportan un Alfa de Cronbach general de $.71 \mathrm{y}$ correlaciones bivariadas entre $.42 \mathrm{y}$ .57 con el resto de componentes de la medida original. Para el presente estudio se obtuvo un Alfa de Cronbach de .79. Según Carlo y Randall (2002), como se esperaba teóricamente, esta medida correlaciona positivamente con variables sociocognitivas como la toma de perspectiva y el razonamiento prosocial interiorizado. Además, no se encontró una asociación significativa con deseabilidad social. 
Medida de tendencias morales de uso de Facebook

Es un instrumento construido para este estudio. Para su construcción se procedió con una etapa inicial de evaluación de ítems por parte de expertos, a partir de la cual se hizo una preselección de los mismos. Posteriormente se valoró la medida recurriendo al procedimiento de la entrevista cognitiva para la evaluación de medidas, de conformidad con lo propuesto por Willis (2005). Dicha entrevista se llevó a cabo con cinco personas, con las mismas características del grupo de estudio definitivo. Como resultado de la entrevista se realizaron cambios, para finalmente obtener la versión utilizada en este estudio. Consta de dos subescalas:

Tendencias de uso hedonista: se dirige a medir el uso orientado al sí-mismo presentando imágenes propias, estados de ánimo o actividades personales en Facebook. Consta de nueve ítems con una escala tipo Likert de siete puntos (totalmente de acuerdo a totalmente en desacuerdo). Ejemplo de ítems: "Publico fotos sobre mí mismo(a)", "Expreso cosas de mi vida privada para que mis contactos sepan cómo me siento". Se obtuvo un coeficiente Alfa de Cronbach de .87 .

Tendencias de uso eudaimónico: el instrumento también fue desarrollado para esta investigación. Mide el uso dirigido a la búsqueda del bien común y la justicia, la expresión de empatía, el respaldo de causas sociales, la denuncia de problemas o el ayudar a amigos que lo requieran. La medida está formada por 17 ítems con una escala tipo Likert de siete puntos (totalmente de acuerdo a totalmente en desacuerdo). Ejemplos de ítems son "Cuando un amigo de Facebook tiene algún problema trato de ayudarlo", "Le doy 'me gusta' a páginas que le interesan mejorar la calidad de vida de las personas en desventaja social". El coeficiente Alfa de Cronbach fue de .89 .

Los ítems de ambas subescalas se presentaron de forma mezclada, para lo cual se distribuyeron de forma aleatoria.

Auto-evaluación de uso autopresentativo: medida de un ítem. Se les solicita calificarse en una escala de 0-10 sobre qué tanto su actividad en Facebook se dirige a expresar o comunicar, imágenes, estados de ánimo o intereses personales.

Auto-evaluación de uso prosocial: medida de un ítem. Se les solicita calificarse en una escala de 0-10 sobre qué tanto su actividad en Facebook se dirige a apoyar a otras personas, causas sociales o ecológicas.

Frecuencia de uso de Facebook: cantidad de horas semanales de uso reportadas. Para medir esta variable se les presentó a las y los participantes un horario semanal, dividido en hora, donde las personas debían de marcar las horas que usan Facebook cada día de la semana.

Para la aplicación del instrumento se recurrió a un cuestionario auto-aplicado que los participantes completaron en el laboratorio de cognición.

Para los análisis se recurrió a la regresión lineal múltiple, método paso por paso. El análisis de regresión múltiple se

Global Media Journal México 15(28). Enero - junio 2018. Pp. 66-77. 
dirige a estudiar las asociaciones entre diferentes variables, permitiendo identificar el efecto de posibles variables, llamadas predictoras o explicativas, sobre una variable dependiente. El tipo de predicción que busca este análisis es de contingencia no es temporal, es decir, si la presencia de una variable contribuye a entender el comportamiento de otra variable, sin que suponga una relación de causalidad (Tabachnick y Fidell, 2007). Se recurrió al paquete estadístico SPSS versión 21 para efectuar los análisis.

\section{Resultados}

Se efectuó un análisis de regresión lineal recurriendo al método stepwise [paso a paso], con el fin de determinar los posibles predictores de las tendencias de uso en estudio (ver tabla 1). Se encontró que las tendencias de uso hedonistas se asocian positivamente con la intensidad de uso de Facebook $(\beta .12, \mathrm{p}<.05)$ y la autoevaluación del uso auto-presentativo $(\beta .68$, $\mathrm{p}<.0001$ ), y negativamente con el altruismo $(\beta-.22, \mathrm{p}<.0001)$. Por su parte, las tendencias de uso eudaimónico se relacionan de forma positiva con la auto-evaluación del uso prosocial $(\beta .73, \mathrm{p}<.0001)$. Estos resultados indican que un uso más intensivo de Facebook se asocia con una mayor presencia de tendencias hedonistas de esta red social. Así mismo, el predominio de dichas tendencias hedonistas se predice por una mayor auto-percepción de uso autopresentativo, pero por puntajes bajos en altruismo. En lo que respecta al predominio de las tendencias eudaimónicas va a ser predicho por puntuaciones altas en la autopercepción de uso prosocial.

Tabla 1. Predictores de las tendencias de uso hedonistas y eudaimónicas

\begin{tabular}{lll}
\hline & Tendencias & Criterios \\
\hline hedonistas & $\beta$ & $\begin{array}{c}\text { Tendencias } \\
\text { eudaimónicas }\end{array}$ \\
\hline Predictores incluidos en el modelo &, $12^{*}$ & $\beta$ \\
\hline Intensidad de Uso de Facebook &, $68^{* *}$ &, $73^{* *}$ \\
\hline Auto-percepción uso auto-presentativo & & \\
\hline Auto-percepción uso prosocial &,$- 22^{* *}$ & \\
\hline Altruismo & &, 55 \\
\hline Ajuste &, 62 & $145,89 * *$ \\
\hline$R^{2}$ & $64,29 * *$ & $(\mathrm{gl} \mathrm{l,121)}$ \\
\hline$F$ & $(\mathrm{gl} \mathrm{3,121)}$ & \\
\hline
\end{tabular}

Fuente elaboración propia

Nota: $* p<.05 . * *<.0001$.

Global Media Journal México 15(28). Enero - junio 2018. Pp. 66-77. 


\section{Discusión}

Con respecto a las hipótesis propuestas, altos puntajes en altruismo no fueron predictores del predominio de tendencias de uso eudaimónicas, como lo presuponía la H1. En cuanto a la $\mathrm{H} 2$ en cambio, se encontraron evidencias empíricas que la respaldan, ya que mayores puntajes de tendencias de uso hedonista se asocian a menores puntajes de altruismo. La H3 presentó evidencias de respaldo empírico parcial, al encontrarse que la tendencia de uso hedonista fue predicha por el uso auto-presentativo, pero no por el prosocial, resultando en que las personas que consideran hacer un uso predominantemente auto-presentativo presentan un uso hedonista de Facebook. Asimismo, la tendencia de uso eudaimónico fue predicho por el uso prosocial, estableciéndose una asociación positiva entre ambas dimensiones, lo que aporta respaldo empírico para la $\mathrm{H} 4$, aunque sin que fuera posible encentrarse una asociación negativa con los usos auto-presentativos.

Además, se encontró que únicamente la tendencia de uso hedonista es predicha por la intensidad de uso de Facebook, variable que fue incluida como variable interviniente en el modelo de regresión, resultando que el uso más intenso de esta red social contribuye a la explicación de la predominancia en el uso hedonista.
Los resultados aquí obtenidos nos ofrecen indicios parciales de la presencia de estas dos tendencias de uso.

Se encontró evidencia empírica que contribuye al estudio de las tendencias hedonistas como vinculadas al interés por mantener una imagen positiva, en la aceptación social y de dar cuenta de símismo ante otras personas, como sucede con su asociación positiva con la autoevaluación del uso auto-presentativo, pero igualmente como distanciadas de conductas altruistas. De igual forma, se encontró evidencia relevante que aporta a la comprensión de la asociación entre las tendencias eudaimónicas y un uso de Facebook dirigido al bienestar de las otras personas como es el caso del uso prosocial, lo cual se podría explicar por las mismas características de las tendencias eudaimónicas, en tanto orientadas a la actualización de los valores propios.

Desde el punto de vista teórico, y considerando los antecedentes empíricos existentes (Tamborini, 2013), el uso de una $\mathrm{u}$ otra tendencia estaría definiendo las relaciones interpersonales, grupales e intergrupales en la red, las actitudes respecto a la red, así como las normas que guían su uso y la relevancia atribuida a los tipos de comportamiento de las otras personas que conforman su red. Así, retomando lo señalado por Weber, Popova y Mangus (2013) de forma hipotética, los resultados encontrados en este estudio podrían sugerir un predominio de mecanismos automáticos, 
es decir, de heurísticos morales, ya sea hedonistas o eudaimónicos que son los que favorecerían el uso auto-presentativo o prosocial. El hecho de que el altruismo se asociara negativamente con las tendencias hedonistas, es un indicio de ello, precisamente porque al estar las conductas altruistas orientadas al bienestar de otras personas y no al mantenimiento de estados de ánimo internos positivos, el heurístico moral implicado se asociaría más bien con las tendencias eudaimónicas. Estas afirmaciones, sin embargo, requieren de mayor investigación, recurriendo más bien a métodos experimentales.

El hecho de que la tendencia de uso hedonista se asocie con la intensidad de uso de Facebook resulta relevante desde el punto de vista teórico. Pareciera que el disfrute asociado a esta tendencia requiere no sólo el realizar acciones auto-presentativas como ya se ha mostrado aquí, sino que estas deben de prolongarse en el tiempo. Este mecanismo parece no estar presente en la tendencia de uso eudaimónico, donde las acciones son las que podrían estar generando el disfrute directo independientemente de la duración de uso. De igual modo, son resultados que requieren de mayor investigación.

Para dar continuidad a esta línea de investigación es importante la realización de estudios experimentales que permitan identificar posibles relaciones causales ente el tipo de tendencia y el comportamiento en las redes sociales informatizadas, ya sea recurriendo a un diseño intrasujeto de medidas repetidas o un diseño de dos grupos experimentales y grupo control. Reto importante resulta aquí, la definición de las tareas experimentales apropiadas de forma que sea posible evaluar más efectivamente los posibles efectos de las dos tendencias sobre conductas o cogniciones relevantes.

\section{Referencias bibliográficas}

Aristóteles (1985). Ética nicomáquea. Ética eudemia. Madrid: Gredos.

Bartsch, A., y Hartmann, T. (2017). The role of cognitive and affective challenge in entertainment experience. Communication Research, 44(1), 29-53.

Bryant, J., Ewoldsen, D., y Cantor, J. (2003). Communication and Emotion. Essays in Honor of Dolf Zillmann. New Jersey: Lawrence Erlbaum.

Carlo, G., y Randall, B. (2002). The development of a measure of prosocial behaviors of late adolescents. Journal of Youth and Adolescence, 31, 31-44.

Eden, A., Grizzard, M., y Lewis, R. (2013). Moral psychology and media theory. Historical and Emerging Viewpoints. En R. Tamborini (Ed). Media and the moral Mind (pp. 26-42). New York: Routledge.

Global Media Journal México 15(28). Enero - junio 2018. Pp. 66-77. 
Gnambs, T., y Appel, M. (2017). Narcissism and social networking behavior: a meta-analysis. Journal of Personality, 86(2), 200-212. https://doi.org/10.1111/jopy.12305

Horton, R., Reid, C., Barber, J., Miracle, J., y Green, J. (2014). An experimental investigation of the influence of agentic and communal Facebook use on grandiose narcissism. Computers in Human Behavior, 35, 93-98.

Lewis, R., Tamborini, R., y Weber, R. (2014). Testing a dual-process model of media enjoyment and appreciation. Journal of Communication, 64, 397-416.

Marshall, T., Lefringhausen, K. y Ferenczi, N. (2015). The big five, self-esteem, and narcissism as predictors of the topics people write about in Facebook status updates. Personality and Individual Differences, 85, 35-40.

Muñiz, J.A. (2015). La comunicación eudaimónica: confluencias entre la comunicación y la felicidad. Communication \& Social Change, 3(1), 48-76. doi:10.17583/csc.2015.1775

Oliver, M., y Bartsch, A. (2010). Appreciation as audience response: Exploring entertainment gratifications beyond hedonism. Human Communication Research, 36, 53-81.

Oliver, M., y Raney, A. (2011). Entertainment as pleasurable and meaningful: Identifying hedonic and eudaimonic motivations for entertainment consumption. Journal of Communication, 61, 984-1004.

Panek, E., Nardis, Y., y Konrath, S. (2013). Mirror or megaphone? How relationships between narcissism and social networking site use differ on Facebook and Twitter. Computers in Human Behavior, 29, 2004-2012.

Reinecke, L., Vorderer, P., y Knop, K. (2014). Entertainment 2.0? The role of intrinsic and extrinsic need satisfaction for the enjoyment of facebook use. Journal of Communication, 64(3), 417-438.

Rivera C., y Garbanzo G. (2010). Uso de un videojuego de simulación digital comercial como herramienta pedagógica en el aprendizaje de la informática en un grupo de adultos del cantón de Curridabat. (Tesis de Licenciatura en Psicología inédita). Universidad de Costa Rica. San José.

Stat Counter. (2018). Recuperado el 28 de mayo, 2018 de http://gs.statcounter.com/\#allsocial_media-CR-yearly-2017-2018-bar

Tabachnick, B. G., y Fidell, L. S. (2007). Using multivariate statistics, $5^{\text {ta }}$ ed. Boston: Pearson. Tamborini, R. (2013). Media and the moral mind. New York. Routledge.

Valenzuela, S., Park, N., y Kee, K. F. (2009), Is there social capital in a social network site? Facebook use and college students' life satisfaction, trust, and participation. Journal of Computer-Mediated Communication, 14,875-901. doi: 10.1111/j.10836101.2009.01474.x

Vorderer, P., Klimmt, Ch., y Ritterfeld, U. (2004). Enjoyment: At the heart of media entertainment. Communication Theory, 14(4), 388-408.

Global Media Journal México 15(28). Enero - junio 2018. Pp. 66-77. 
Vorderer, P. (2011). What's next? Remarks on the current vitalization of entertainment theory. Journal of Media Psychology, 23, 60-63. doi:10.1027/1864-1105/a000034

Vorderer, P. y Reinecke, L. (2015). From Mood to Meaning: The Changing Model of the User in Entertainment Research. Journal of Communication, 25(4), 447-453.

Weber, R., Popova, L., y Mangus, M. (2013). Universal morality, mediated narratives and neural synchrony. En R. Tamborini (Ed). Media and the moral mind. (pp. 26-42). New York: Routledge.

Willis, G.B. (2005). Cognitive interviewing: A tool for improving questionnaire design. Thousand Oaks, CA: Sage Publications.

Wirth, W., Hofer, M., y Schramm, H. (2012). Beyond pleasure: Exploring the eudaimonic entertainment experience. Human Communication Research, 38, 406-428.

Verduyn, P., Ybarra, O., Résibois, M., Jonides, J., y Kross, E. (2017). Do social network sites enhance or undermine subjective well-being? A critical review. Social Issues and Policy Review, 11(1), 274-302.

Zillmann, D. (1988). Mood management through communication choices. American Behavioral Scientist, 31, 327-340.

Global Media Journal México 15(28). Enero - junio 2018. Pp. 66-77. 